\title{
Mitochondrial Membranes Restitution Proceeds via Vesicular Import from ER and Cytosol. Counterparts' Resemblances and Variances in Mitochondria and Golgi Pathways
}

\author{
Amalia Slomiany, Bronislaw L. Slomiany \\ Rutgers, Biomedical and Health Sciences, School of Dental Medicine, Newark, USA \\ Email: slomiaam@sdm.rutgers.edu
}

How to cite this paper: Slomiany, A. and Slomiany, B.L. (2017) Mitochondrial Membranes Restitution Proceeds via Vesicular Import from ER and Cytosol. Counterparts' Resemblances and Variances in Mitochondria and Golgi Pathways. Advances in Biological Chemistry, 7, 1-26. https://doi.org/10.4236/abc.2017.71001

Received: January 6, 2017

Accepted: February 5, 2017

Published: February 8, 2017

Copyright $\odot 2017$ by authors and Scientific Research Publishing Inc. This work is licensed under the Creative Commons Attribution International License (CC BY 4.0).

http://creativecommons.org/licenses/by/4.0/

\section{(c) (i) Open Access}

\begin{abstract}
The processes of mitochondrial restitution are controlled by nuclear genes that encode proteins synthesized in ER and cytosol and delivered as organelleand membrane-specific transport vesicles. The analysis of the transporters recovered from inner mitochondrial space (Mitosol) revealed that the ERsynthesized mitochondria-specific transport vesicles consist of two carriers, one remaining in outer mitochondrial membrane (OMM), and the other that transfers specific membrane segments to the inner mitochondrial membrane (IMM). The ER-assembled and IMM-committed membrane segments, while first integrated into OMM, undergo intra-mitochondrial lipid modification reflected in the synthesis of cardiolipin (CL) and inversion into Mitosol with load of IMM associated cytosolic proteins. Then, the CL-bedecked vesicles are released from OMM to Mitosol and upon contact with IMM fuse with the membrane, and the release of cytosolic cargo ensues. While ER-assembled mitochondria-specific transport vesicles fuse with OMM with the aid of the cytosolic, phosphatidylglycerol (PG)-specific phospholipase $\mathrm{A}_{2}\left(\mathrm{PLA}_{2}\right)$, the Mitosol-contained CL-specific PLA guides vesicles fusion with IMM. The described path of translocation of the membrane segments and the cytosol synthesized proteins into the designated mitochondrial compartments sustains growth and identity of OMM, IMM, maintains protein delivery for intramitochondrial lipid and protein modification in Mitosol, and ensures conformity of the cytosolic proteins cargo delivered to matrix.
\end{abstract}

\section{Keywords}

Mitochondrial Restitution, ER Vesicular Transport, Mitochondrial

Membranes, Cardiolipin, Mitosol Vesicles 


\section{Introduction}

In line with the concept of cell organelles discrete specificity, the fidelity of their restitution and accuracy in retaining their function, are most essential and command processes that are exquisite for every cellular compartment [1]-[9]. This poses fastidious tasks on trafficking of the newly synthesized replacements to their exact sites and especially presents incredibly difficult challenge for the import of genome encoded ER- and cytosol-residing components to mitochondria [3] [5] [6] [7] [10] [11] [12]. Consequently, numerous pathways for protein trafficking and translocation across the organelle's membranes are described [10] [13]-[24]. Yet, the molecular mechanisms that distinguish different pathways still require deeper exploration, and above all, the investigation of the role of lipids in controlling and discerning these processes [2]-[7] [10] [25] [26] [27]. The features of the currently advocated import pathways stipulate the recognition of the cellular location mediated by the import apparatus, recognition of the docking sites for the incoming replacement elements, generation of translocation channels by incoming membrane associated protein, and conformity of the transferred components [28]. Although, theoretically these features fulfill exquisite demands regarding import devices, the final probes tailored exclusively from the outer- or inner mitochondrial membrane (OMM and IMM, respectively) proteins without the membrane's-specific lipids, their insertion into proper site of the membrane and the in situ function are doubtful. Without native, cotranslationally introduced lipids, the authenticity of protein configurations is not reproduced and the reliability of the transfer into the precise site that determine membranes function remain ambiguous [28]-[34]. In case of IMM restitution, the so known "translocases" of the inner membrane (TIM) must travers OMM, navigate through Mitosol and precisely intercalate the membrane complexes into the specific site of inner membrane. The doubt provoking are the stipulated intricate steps that are not based on observable facts, while inferring on the differences in protein destination, their biosynthetic derivation and factual affinity. The possibility of their promiscuity that would prompt or halt their interaction with OMM, or induce their transit and elicit interaction with IMM, all the while carrying the cytosolic precursors to the existing membrane and matrix is not contemplated [28] [29] [35] [36]. Moreover and again, the concept of engaging the translocase protein sets, which are not shielded from mixture of other cytosol genomic products, assembled for similar but not for identical sites, raises concern whether the precision required for the translocation of the substrates is achieved.

As mentioned earlier, the import pathways described so far are missing two major elements; first how the cores of mitochondrial membranes are restored by the nuclear genome-synthesized protein products, and the second how and when the membrane-specific protein complexes are intercalated into specific lipid combinations required for complete, unchanged and undisturbed assemblies of OMM and IMM [27]. While the importance of lipids in mitochondrial protein import is the recently emerging topic of investigation, the current models 
are based on the assumption that all membranes are identical and formed through the incorporation of individual lipids and proteins into existing cellular prototypes [28] [37] [38] [39].

The speculative machineries, described as translocases of outer membrane (TOM) and the translocases of inner membrane (TIM) composed of protein complexes and individually introduced lipids released into cytosolic soluble mixture, could not result in consistently precise intercalation of protein, and would fail to restore the organelle structure, composition and its function. Again, the above described mechanism of organelles restoration is not considering the structural requirements of lipids in OMM and IMM and thus provides sketchy and inaccurate picture [28] [40]. Another concept of mitochondrial restitution envisages transport between ER and mitochondria as contact sites in form of ER-mitochondrial tunnels, but that cannot explain the intramitochondrial transport of cytosolic protein, and probably reflects the presence of cocreated autosomal formations [6] [33] [41]. Hence the described ideas, supported by arbitrary choice of steps in the delivery of the protein complexes, create further perplexity in mitochondrial membranes restoration and the cytosolic proteins transit through OMM, Mitosol, and IMM to reach the matrix. Consequently, it is appropriate to state that in spite of numerous explanation rendered for interorganellar (between organelles) and intraorganellar (within organelle) protein and lipid transport, the biogenesis and the restitution of mitochondrial membranes is not explained.

The criteria that satisfy the requirements of membrane make up fidelity and the deposition of organelle specific proteins were systematically investigated in our work [1]-[7] [36] [42] [43] [44] [45]. Coherent picture derived from the multiplicity of experiments revealed that the only process that fulfills these principle requirements is ER-localized biogenesis of the genomically-defined membrane proteins cotranslationally intercalated within explicit set of co-synthesized lipids that together form a distinct, organelle-precise components in a form of transport vesicles. Thus, based on our extensive study into cellular vesicular transport, we strongly endorse the concept that the delivery and restoration of the membranes, as well as transfer of ER-synthesized cargo can only be accomplished with the aid of precisely constructed transport vesicles which through affinity to proper site are capable to restore genome-controlled cell rebuilding [1] [2] [3] [4] [5] [7] [36] [45].

Based on the concept described, supported by the vesicular lipid analyses of the Golgi-directed transporters, we validated the process of mitochondrial restitution via vesicular import of nuclear products to OMM and Mitosol [5]. We have demonstrated that the mitochondria-specific ER-assembled transport vesicles are formed and are substantially dissimilar from those generated for restitution of Golgi, endosomes and cell membrane [3] [5] [7]. The major differences in membrane lipid composition found in vesicles destined to mitochondria is the presence of phosphatidylglycerol (PG), the absence of sphingolipids (SphL) and phosphatidylinositol (PI), and that their fusion with OMM is accompanied by 
generation of lysophosphatidylglycerol (LPG). However, the recognition of the initial step in mitochondrial membranes restitution has not offered the explanation as to the processing of IMM and the delivery of mitochondria-dedicated cytosolic constituents [11] [28] [38] [40].

The aim of the herein described investigations is to evaluate working hypothesis that the restitution of IMM and transfer of cytosolic proteins into matrix are accomplished through OMM-inserted but IMM-specific vesicles, which at that stage become packed with cytosolic cargo interacting with IMM-specific membrane, and through invagination and sealing thenceforward the vesicles enter Mitosol, and upon contact with IMM fuse with its membrane and release its cytosolic cargo to matrix. The invagination process is associated with the formation of cardiolipin $(\mathrm{CL})$, whereas the fusion with IMM by the generation of lysocardiolipins (LCLs). Our investigations support the outlined mode of OMM platform transferring maternal ER membrane to IMM while delivering the cytosolic proteins to matrix. Moreover, in principle, the restorative processes of mitochondria appear similar with Golgi pathway, a conduit dedicated to renew Golgi membranes, endosomes, and cell membranes [5] [7] [25] [36] [43] [46].

Thus, it is our evidence-supported conclusion that the factors that initiate synthesis of cell and its organelles, including mitochondria, and those impacting synthesis of cytosolic proteins are intimately entwined and produce response that effectively delivers products to the correct destination. These highly specialized connections culminate in a precise organelle's membranes synthesis only when series of genome-initiated actions and co-regulated compartmentalized lipids synthesis in ER are upheld.

\section{Materials and Methods}

\subsection{Subcellular Fractionation}

Fragmentation of cell structure aiming at the isolation of intact organelles is crucial and highly critical step that should produce minimum breakage of membrane-surrounded components differing in size, composition and buoyancy. With this in mind, the methods described here have been developed and modified to obtain mitochondria with minimum breakage so that Cell Cytosol (CC) was not admixed with organellar components and that intact organelle could be used for the isolation of Outer Mitochondrial Membrane (OMM), Inner Mitochondrial Membrane (IMM) and the inner mitochondrial space content referred to as Mitosol. Briefly, the cells were prepared from rat liver as described previously [1] [4] [5] [25] [43]. The single cells that were separated from larger debris with aid of specific cell size nylon mesh were centrifuged at $50 \mathrm{xg}$ for $2 \mathrm{~min}$, washed twice with the enzyme-free medium, twice with the Minimum Essential Medium (MEM) and counted in hemocytometer. Thus prepared cells were then incubated in MEM for 3 hours with or without radiolabel and used for preparation of nuclei, subcellular organelles, cell cytosol and cellular membranes [2] [46] [47]. In the experiments dedicated to the determination of lipid synthesis with cell cytosol derived from untreated or RNase-treated cytosol, the prepara- 
tions of nuclei, mitochondria, ER, Golgi or other organelles and cell membranes were additionally rinsed with phosphate buffered saline (PBS) and 2M urea-PBS in order to remove the associated residual cytosolic proteins that otherwise would remain on the membranes.

Thus prepared subcellular organelles and membranes were used for experiments on transport vesicles synthesis [2] [3] [5] [7] [46] and the preparation of OMM and IMM [5] [48]. The synthesis of phospholipids and protein was determined using radiolabeled $\left[{ }^{3} \mathrm{H}\right]$ inositol, $\left[{ }^{3} \mathrm{H}\right]$ arachidonate, $\left[{ }^{3} \mathrm{H}\right]$ choline, $\left[{ }^{3} \mathrm{H}\right]$ serine, $\left[{ }^{3} \mathrm{H}\right]$ palmitate and $\left[{ }^{32} \mathrm{P}\right] \mathrm{ATP}$ [1] [3] [4] [25] [43] [46]. The radiolabeled ER transport vesicles fusion with Golgi and then mitochondria were performed in medium containing cold cell cytosol (CC) at concentration of $15 \mathrm{mg}$ protein $/ \mathrm{ml}$ of incubation mixture enriched with $50 \mu \mathrm{M}$ ATP, $250 \mu \mathrm{M}$ CTP, $50 \mu \mathrm{M}$ GTP, $5 \mathrm{mM}$ creatine phosphate, $8.0 \mathrm{IU} / \mathrm{ml}$ creatine kinase, and where indicated $25 \mu \mathrm{g} / \mathrm{ml}$ RNase, $10 \mu \mathrm{M}$ UDP-Glc and $10 \mu \mathrm{M}$ palmitoyl CoA [1] [3] [4] [25] [43] [46].

\subsection{Preparation of Transport-Active Cell Cytosol (CC)}

The viable cells, homogenized for $10 \mathrm{sec}$ at $600 \mathrm{rpm}$ in 3 volumes of buffer containing $0.25 \mathrm{M}$ sucrose; $50 \mathrm{mM}$ TRIS- $\mathrm{HCl}$ ( $\mathrm{pH}$ 7.4), $25 \mathrm{mM}$ magnesium acetate and $10 \mathrm{mM}$ each of aprotinin, leupeptin, chemostatin, and $1 \mathrm{mM}$ phenylmethylsulfonylfluoride, were centrifuged at $5000 \mathrm{xg}$ for $15 \mathrm{~min}$. The supernatant, diluted with 2 volumes of homogenization buffer, was re-centrifuged at 10,000 $\mathrm{xg}$ for $20 \mathrm{~min}$. The resulting supernatant was then subjected to centrifugation at $100,000 \mathrm{xg}$ for $1 \mathrm{~h}$. Thus obtained soluble fraction was adjusted to $15-18 \mathrm{mg}$ protein $/ \mathrm{ml}$, admixed with an ATP generating system consisting of $40 \mathrm{mM}$ ATP, $200 \mathrm{mM}$ creatine phosphate, $2000 \mathrm{units} / \mathrm{ml}$ creatine phosphokinase, and referred to as transport active cell cytosol or active cytosol (CC).

\subsection{Preparation of Cellular Organelles and Membranes}

The cell membranes and subcellular organelles (mitochondria, ER, Golgi) were recovered from cold or radiolabeled cells as described earlier [1] [3] [4] [5] [25] [43] [46]. The ER and Golgi organelles sediment, remaining after separation of nuclei, mitochondria and cell cytosol, was suspended in buffer containing $0.2 \mathrm{M}$ PIPES (pH 6.9), $2 \mathrm{M}$ glycerol, $1 \mathrm{mM}$ EGTA and $1 \mathrm{mM}$ magnesium acetate and applied on the top of discontinuous gradient of 2.0/1.5/1.3/1.0 M sucrose and centrifuged at 100,000 $\mathrm{xg}$ for $16 \mathrm{~h}$. The cell membranes were recovered from 1.0 M sucrose, Smooth Endoplasmic Reticulum (SER) from 1.3 M sucrose, RER from 1.5 M sucrose and Golgi from the top of the 2.0 M sucrose. Each sucroseseparated fraction was subjected to further purification. The cell membranes were washed with original PIPES buffer and centrifuged at $3000 \mathrm{rpm}$ for $2 \mathrm{~min}$. To separate cells' membrane, the buffer was adjusted with $0.2 \%$ Triton X-100 and the mixture incubated at $4^{\circ} \mathrm{C}$ for $5 \mathrm{~min}$ [3] [46]. This treatment resulted in breaking up the phosphoglycerides-rich membranes into smaller segments and thus allow us to separate membranes containing cholesterol, sphingolipids and 
glycoproteins. The latter membranes were recovered by low speed centrifugation at $3000 \mathrm{rpm}$ for $2 \mathrm{~min}$.

\subsection{Generation and Purification of Transport Vesicles}

ER-derived transport vesicles were generated in the presence of radiolabeled precursors according to procedure described previously [1] [3] [4] [7] [10] [11] and where indicated, in the presence of Brefeldin A (BFA) [5] [6] [44]. The ER mixed with cytosol, ATP-generating system, UTP, CTP GTP, fatty acyl CoA and water soluble cold or radiolabeled lipids precursors, were incubated for $30 \mathrm{~min}$ at $37^{\circ} \mathrm{C}$, centrifuged over $0.3 \mathrm{M}$ sucrose and treated with stripping buffer at $2^{\circ} \mathrm{C}$ for $15 \mathrm{~min}$ followed by centrifugation at $10,000 \mathrm{xg}$ for $10 \mathrm{~min}$ to separate transport vesicles from ER or Golgi membranes. The separated from maternal membranes transport vesicles were recovered from the supernatant resulting from centrifugation of the supernatant mixture at 150,000 xg for $60 \mathrm{~min}$. The crude fraction of the transport vesicles was suspended in 55\% sucrose, overlaid with $55 \%-30 \%$ gradient and centrifuged at $150,000 \mathrm{xg}$ for $16 \mathrm{~h}$. The purified transport vesicles were recovered from the gradients as reported earlier [1] [3] [4] [25] [43] [46].

\subsection{Separation of ER-Mitochondrial and ER-Golgi Vesicles by Fusion of ER-Derived Transport Vesicles with Golgi [5]}

One volume of ER transport vesicles (1.3 - $1.5 \mathrm{mg}$ protein/ml) was suspended in one volume of active cytosol ( $15 \mathrm{mg}$ protein $/ \mathrm{ml}$ ), and added to one volume of cell organelles ( $5 \mathrm{mg}$ protein $/ \mathrm{ml}$ ). The reaction was allowed to proceed from 0 $30 \mathrm{~min}$ at $4^{\circ} \mathrm{C}$ (control) and at $37^{\circ} \mathrm{C}$ in the presence of ATP regenerating system consisting of $40 \mathrm{mM} \mathrm{ATP}, 200 \mathrm{mM}$ creatine phosphate, $2000 \mathrm{units} / \mathrm{ml}$ of creatine phosphokinase, or in the ATP depleting system containing $5 \mathrm{mM}$ glucose and 500 units/ml hexokinase. After incubation, the Golgi organelles were recovered by centrifugation through three volumes of $0.5 \mathrm{M}$ sucrose at $3000 \mathrm{rpm}$ for 5 min. The remaining vesicles recovered from the supernatant after incubation with Golgi were used in the fusion experiments with mitochondria. One volume of the recovered vesicles $(0.9-1.1 \mathrm{mg} / \mathrm{ml})$ was resuspended in one volume of CC $(15 \mathrm{mg} / \mathrm{ml})$ and added to one volume of purified mitochondria $(5 \mathrm{mg} / \mathrm{ml})$ and the reaction was allowed to proceed for $30 \mathrm{~min}$ under same conditions as described for the ER vesicles fusion with Golgi. In the experiments estimating en bloc fusion of transport vesicles with Golgi and/or mitochondria, the associated but not fused vesicles were released from the membrane by subjecting the membrane fraction to treatment with $2 \mathrm{M}$ urea at $4^{\circ} \mathrm{C}$ and then the recovered organelles were centrifuged through $0.5 \mathrm{M}$ sucrose, washed and subjected to lipid analysis.

\subsection{Isolation and Purification of Mitochondrial Membranes [5]}

The mitochondria were isolated from the hepatocytes derived from perfused rat livers subjected to subcellular fractionation that afforded preparation of nuclei, 
mitochondria, Golgi, ER and organelle-free cell cytosol as described in above. From the crude fraction the fluffy layer of broken mitochondria and ER microsomes that covered the undamaged mitochondrial pellet was suctioned off, the pellet was gently resuspended in five volumes of medium consisting of $0.07 \mathrm{M}$ sucrose, $0.21 \mathrm{M}$ mannitol, $0.1 \mathrm{mM}$ disodium EDTA, and $1 \mathrm{mM}$ TRIS, pH 7.2. and the suspension was spun for $10 \mathrm{~min}$ at low speed $(500 \mathrm{xg})$, the supernatant recovered and spun for $10 \mathrm{~min}$ at $9000 \mathrm{xg}$ [5] [48]. This manipulation was repeated three times. Thus purified preparation of mitochondria containing only occasional broken mitochondria, ER membranes, or lysosomes was used for breaking and detaching the Outer Mitochondrial Membrane (OMM). The mitochondria were swollen for $20 \mathrm{~min}$ at $4^{\circ} \mathrm{C}$ in $20 \mathrm{ml}$ of swelling medium $(20 \mathrm{mM}$ phosphate buffer, $\mathrm{pH}$ 7.2). The swelling process was initiated gradually by suspending the pellet in very small amount of swelling medium and progressively, over 3 - $5 \mathrm{~min}$, the mixture diluted to final volume. After $20 \mathrm{~min}$ of the treatment, the preparation was spun for $20 \mathrm{~min}$ at $17,000 \mathrm{rpm}(35,000 \mathrm{xg})$, the pellet and the supernatant were separately recovered for further purification. Moreover, the supernatant containing Mitosol was centrifuged at 100,000 xg, filtered and subjected to centrifugation at 150,000 $\mathrm{xg}$ for $60 \mathrm{~min}$, in the similar manner as in preparation of vesicles-free CC. The Mitosol-derived pellet was then suspended in 55\% sucrose, overlaid with 55\% - 30\% sucrose gradient and centrifuged at 15,000 $\mathrm{xg}$ for $16 \mathrm{~h}$. The Mitosol-derived vesicles were recovered in the same manner as the ER-derived present in the cytosol [1] [2] [5]. The Outer and Inner Mitochondrial Membranes (OMM and IMM)-enriched fraction collected after initial separation of Mitosol was resuspended in fresh swelling buffer containing $0.02 \%$ albumin, and sedimented at $4000 \mathrm{rpm}(1900 \mathrm{xg})$ for $15 \mathrm{~min}$. The OMM and IMM contained in the supernatant were concentrated by spinning at $17,000 \mathrm{xg}$, the pellet resuspended in $4.0 \mathrm{ml}$ of $20 \mathrm{mM}$ phosphate buffer, $\mathrm{pH} 7.2$ and subjected to discontinuous sucrose gradient fractionation.

\subsection{Discontinuous Sucrose Gradient to Separate OMM and IMM}

A three layered gradient was formed in Beckman SW39 tubes by layering successively sucrose solutions prepared in $20 \mathrm{mM}$ phosphate buffer $\mathrm{pH} 7.2$ consisting of $1.2 \mathrm{ml}$ of $51.3 \mathrm{~g} / 100 \mathrm{ml}$ sucrose solution (density 1.142 ), $1.2 \mathrm{ml}$ of 37.7 $\mathrm{g} / 100 \mathrm{ml}$ sucrose (density 1.142 ) and $1.2 \mathrm{ml}$ of $25.2 \mathrm{~g} / 100 \mathrm{ml}$ sucrose (density 1.094). One $\mathrm{ml}$ of concentrated $17,000 \mathrm{xg}$ pellet was layered on the top of the gradient and centrifuged at $37,500 \mathrm{rpm}(115,000 \mathrm{xg})$ for one hour. This resulted in separation of the membranes into three bands. From the top to bottom, the bands consisted of OMM small fragments (A), large sheets of OMM (B) and IMM with some fragments representing OMM (C), respectively. The identification of the isolated fraction is based on the electron microscopic studies published earlier [5] [48]. To remove sucrose, the separated fractions were diluted in $20 \mathrm{mM}$ phosphate buffer, $\mathrm{pH} 7.2$ and centrifuged at 17,000 rpm $(35,000 \mathrm{xg})$ for one hour. Then the $\mathrm{A}, \mathrm{B}$, and $\mathrm{C}$ fractions recovered from discontinuous sucrose gradient were resuspended in $0.5 \mathrm{ml}$ of the same buffer and used for enzyme as- 
says and lipid analysis. The assays were performed in the presence of the Mitosol or the CC components.

\section{Results}

The presented results embody continuation of the investigations into transport of mitochondria-destined proteins encoded by nuclear genes and consequently synthesized as proteins intercalated into endoplasmic reticulum (ER) membranes [2] [5], and as translation products of cytosolic ribosomes [29]. The transport of ER-translated products described previously was found to be promoted by mitochondria-specific vesicles whose membranes consisted of phosphatidylcholine (PC), phosphatidylethanolamine (PE), phosphatidylglycerol (PG) and phosphatidic acid (PA), but free of sphingolipids (SphLs) and phosphatidylinositols (PIs) [5] [7] [25] [42]. The fusion process of the ER-mitochondrial transport vesicles with the outer mitochondrial membrane has not generated any changes in OMM lipid composition, and as illustrated earlier [5] the OMM after fusion with ER-mitochondrial vesicles, consisted of PC, PE, PA, PG and traces of phosphatidylglycerol phosphate (PGP). However, while the vesicular transport event explained growth and restoration of the OMM and delivery of the ER-synthesized protein cargo to Mitosol (the sol specific to inner mitochondrial space, as oppose to cytosol, the sol of the cell), the restoration of the inner mitochondrial membrane (IMM) and transport of proteins synthesized on cytosolic ribosomes remained unexplained.

Based on the paradigm used, and the evidence obtained from the investigations of Golgi-directed transport vesicles that represent assortment of specific products that restore Golgi, endosome and cell membrane [2]-[7], henceforth, we speculated that mitochondria-directed transporters must consist of the vesicles assemblies dedicated for the restoration of OMM, IMM and, that IMMbound transporters carry cytosolic proteins for mitochondrial matrix. Moreover, as our concept and the results dictated, the synthesis of OMM and IMM-directed transport vesicles although initially displaying same lipid composition must be initiated through different signals since their protein composition is not identical, the IMM is richer in protein than OMM and is enriched in CL [5]. Thus, to fulfill these requirements the ER-mitochondrial transport vesicles must consist of the specific ER products for OMM restitution and contain the ERassembled cargo to be released to Mitosol while those for IMM, in addition to inner membrane restitution task, should function as transporters of cytosolic proteins for matrix. Hence, just as cytosol obtains ER-assembled vesicles for all cellular restorations, the Mitosol must receive its quota for IMM refurbishment.

As described in Materials and Methods, the Mitosol, recovered from unlabeled mitochondria used in fusion experiments with $\left[{ }^{3} \mathrm{H}\right]$ palmitate labeled ER transport vesicles was subjected to high speed centrifugation, and the pelleted material following sucrose gradient centrifugation was analyzed (Figure 1).

The Mitosol material that displayed gradient characteristics similar to ERmitochondria specific-transport vesicles labeled with $\left[{ }^{14} \mathrm{C}\right]$ choline was found to 
contain the radiolabel represented exclusively by PC (Figure 2).

The labeling of ER transport vesicles with $\left[{ }^{3} \mathrm{H}\right]$ palmitate revealed the presence of the mitochondria-specific phospholipids identified earlier [5] and defined above, whereas the Mitosol's-the intra-mitochondrial vesicular components were augmented with CL and the lyso derivatives represented by LCL and LLCL were identified in IMM (Figure 3).

The attributes of the ER mitochondrial vesicles and those isolated from Mitosol signaled that the ER-mitochondrial transport vesicles consist of two specific transporters that initially fuse with OMM and release their ER-synthesized cargo to Mitosol. Then, while OMM specific portion of the vesicles remains and restores OMM with lipid profile same as ER-mitochondrial vesicles (Figure 3(a), Figure 3(b)), the IMM specific fragments become augmented with Mitosoldriven CL synthesis, reform into Mitosol-specific transport vesicles (Figure $3(c)$ ) that fuse with IMM and thus restore its CL (Figure 3(d)). Since incorporation of the Mitosol vesicles membrane into IMM is accompanied by the formation of LCL (Figure 3(d)) we conclude that as in OMM, the fusion of ER vesicles generated LPG [5], the process that introduces newly synthesized CL-containing membranes into IMM creates LCL while exposing cytosol-synthesized cargo to matrix.

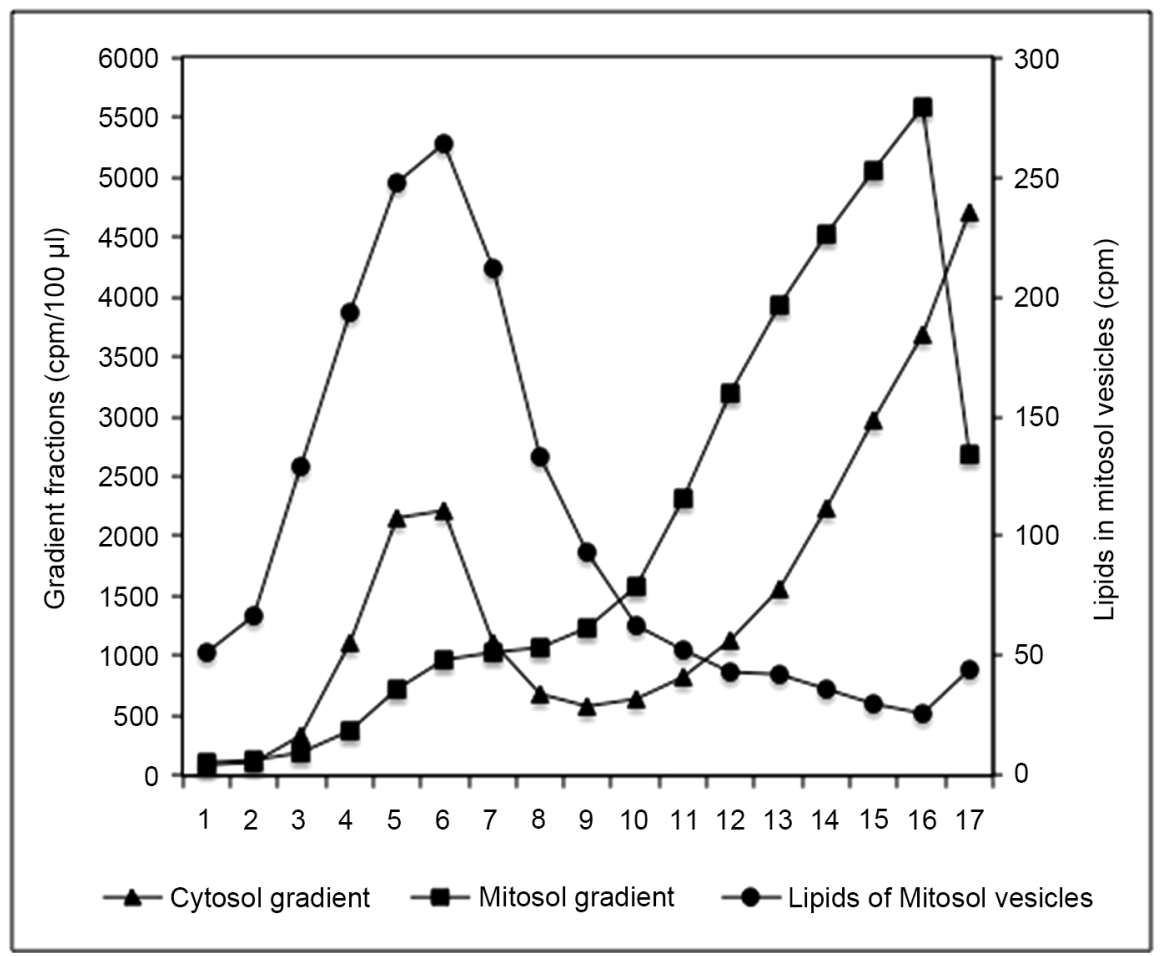

Figure 1. Transport vesicles recovered from cell Cytosol and Mitosol-the mitochondrial inner space sol. The labeling pattern of the Cytosol and Mitosol gradient fractions is depicted on the left panel, whereas the radiolabel extracted with Mitosol vesicles lipids is depicted in the right panel. As illustrated, the Mitosol constituents with sedimentation properties corresponding to vesicular fraction identified in Cytosol contained radiolabeled lipids derived from $\left[{ }^{3} \mathrm{H}\right]$ palmitate introduced to ER-mitochondrial transport vesicles. 


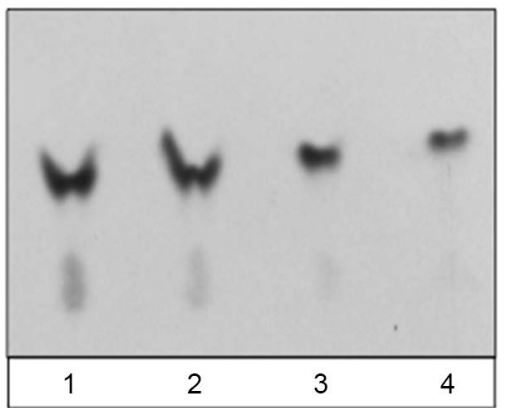

Figure 2. Transfer of $\left[{ }^{14} \mathrm{C}\right]$ choline radiolabel from ER-mitochondrial transport vesicles to Mitosol vesicles and to IMM in form of PC. The lipid extracts correspond to (1) gradient purified ER-mitochondrial transport vesicles, (2) intact mitochondria following incubation with ER-mitochondrial transport vesicles, (3) Mitosol vesicles isolated from mitochondria subjected to incubation with labeled ER-mitochondrial vesicles, (4) IMM lipids labeling in the mitochondria subjected to incubation with labeled ER-mitochondrial vesicles. The radiolabeled spots particularly visible in (1) and (2) reflect the presence of $\left[{ }^{14} \mathrm{C}\right]$ choline label co-extracted with lipids. The possibility that the spots reflect presence of lysophosphatidylcholine (LPC) was eliminated by the determination of lytic activity in ER-mitochondrial vesicles and OMM demonstrated in Figure 7.

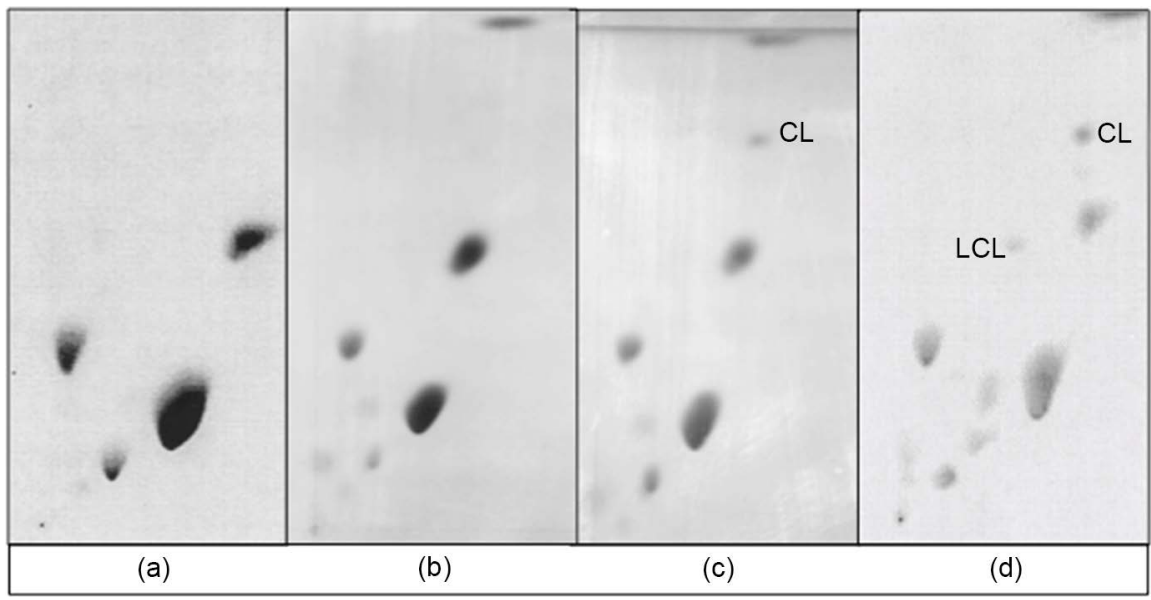

Figure 3. Labeling of ER-mitochondrial transport vesicles with $\left[{ }^{3} \mathrm{H}\right]$ palmitate and transfer of the radiolabeled membrane into mitochondrial membranes and Mitosol vesicles. Panel (a) depicts lipid composition of ER-mitochondrial transport vesicles, panel (b) depicts radiolabeled lipids integrated into OMM, panel (c) shows lipids isolated from vesicles identified in Mitosol and panel D reflects incorporation of radiolabeled lipids derived from ER-mitochondrial transport vesicles in IMM. As pointed out in panel (c) the lipids of Mitosol-derived transport vesicles are enriched with CL, whereas those identified in IMM reflect presence of CL, lysocardiolipin (LCL) and possibly bis-lysocardiolipin (LLCL) which migrates parallel to PC. The presence of LLCL in IMM is further documented in Figure 7 where the possibility of the LPG overlap (panel (b)) is eliminated by finding that Mitosol and IMM are lacking PG-specific phospholipase.

To test such chain of events, we have designed experiments with unlabeled ER-mitochondrial vesicles incubated with unlabeled mitochondria in the presence of $\left[{ }^{3} \mathrm{H}\right]$-serine-labeled cytosol and then investigated the contents of Mitosol transport vesicles for the presence of radiolabeled proteins. As shown in Figure 4 , the $\left[{ }^{3} \mathrm{H}\right]$ serine-labeled cytosolic proteins were identified in Mitosol vesicles, 
thus indicating that these ER-derived transporters after initial fusion with OMM were reloaded with cytosol-derived cargo and released to Mitosol.

As presented in Figure 3 and Figure 4, the vesicles after enrichment with CL fused with IMM, since radiolabeled membrane lipids containing CL and LCL were identified in IMM. Thus, as in previous investigations, the appearance of lysophospholipids following incorporation of radiolabeled vesicles into substrate membrane was indicative of membranes' fusion and the release of their transport cargo [42] [46].

Our concept that membrane rupture and fusion is preceded by PLA-induced formation of the specific lysophospholipids was documented earlier [5] [46] [49]. And, the detergent-like qualities of lysophosphatidylcholine (LPC) in erythrocytes hemolysis is a classical example of the properties common to all lysophospholipids. With these characteristics in mind, the experiment on the opening of Mitosol vesicles using lysocardiolipin (LCL) identified in IMM (Figure 3(d)) was performed (Figure 5). The choline-labeled Mitosol vesicles were incubated with LCL extracted from IMM and the products subjected to sucrose gradient centrifugation.

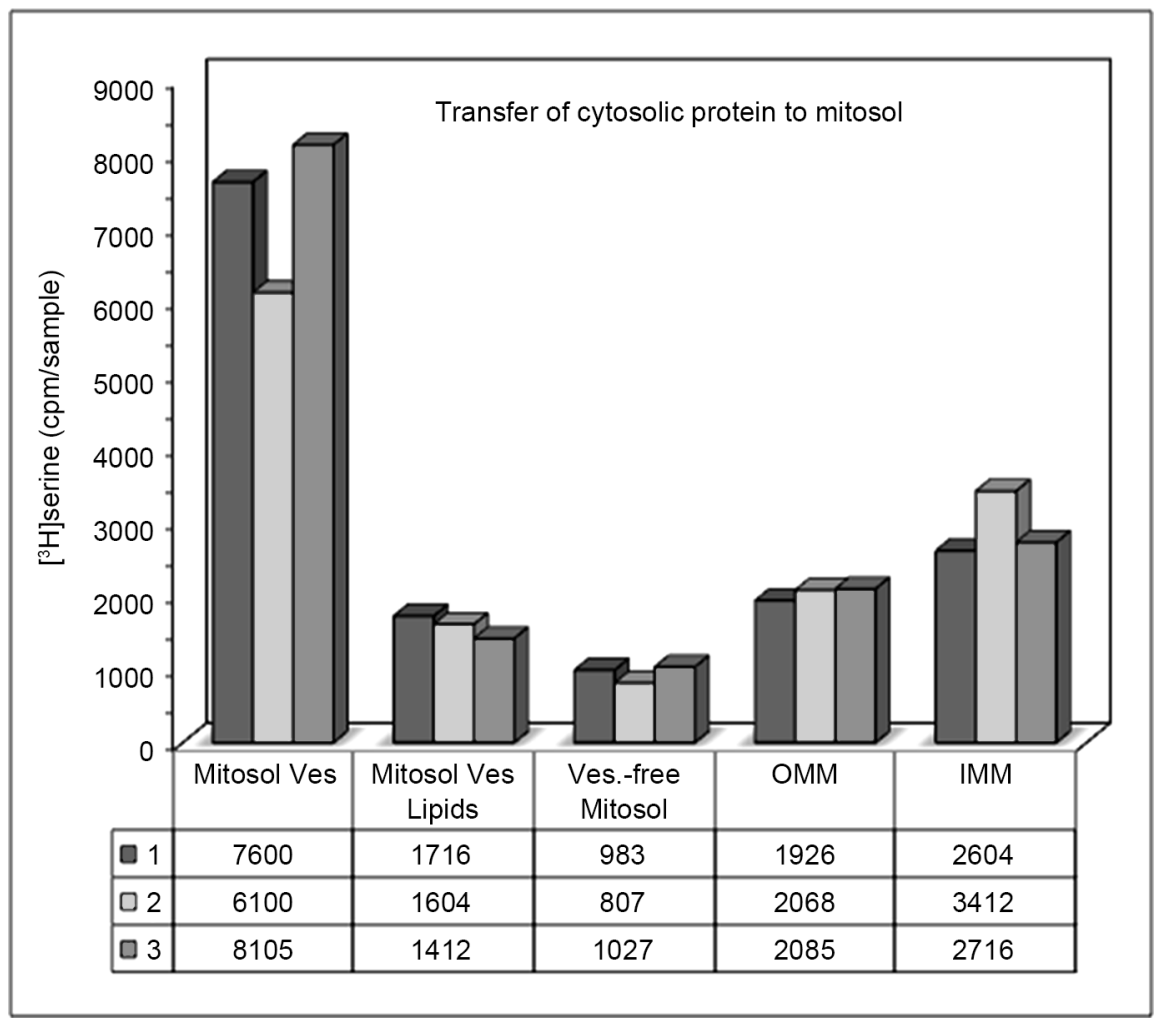

Figure 4. The transport of cytosolic proteins into mitochondria involves ER-produced IMM specific mitochondrial vesicles. As depicted above, the experiment on the ERmitochondrial transport was performed using $\left[{ }^{3} \mathrm{H}\right]$ serine labeled cytosol, and label free ER-mitochondrial vesicles and mitochondria. After an incubation period, used in all experiments resulting in the vesicles fusion with mitochondria, the Mitosol vesicles and the depicted fraction of mitochondria were checked for the presence of labeled cytosolderived components. Three independent experiments, depicted above, demonstrate that Mitosol vesicles contain cytosol protein cargo. 


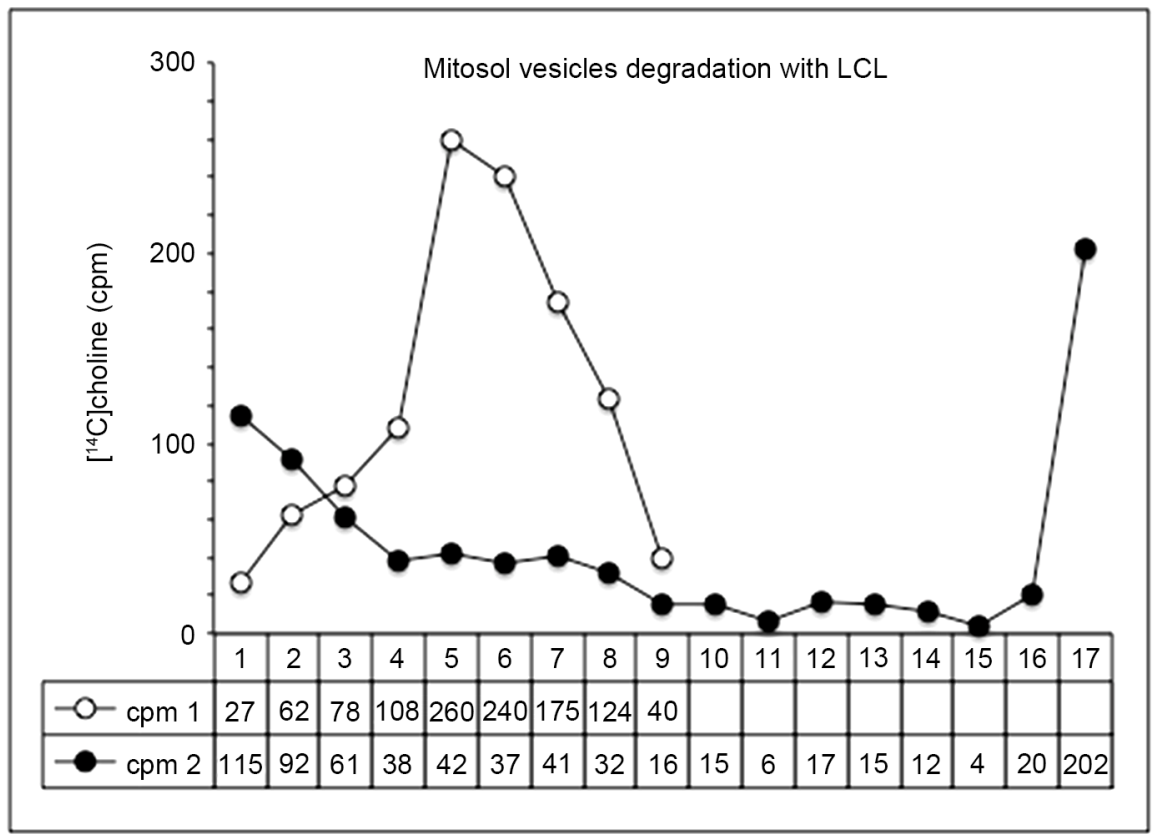

Figure 5. Results of an exposure of $\left[{ }^{14} \mathrm{C}\right]$ choline labeled Mitosol vesicles to LCL extracted from IMM. As illustrated, the aliquots of Mitosol vesicles, incubated in medium free of LCL (cpm 1) and with LCL derived from IMM (cpm 2) were subjected to gradient centrifugation and its fraction examined. Following the incubation with LCL, the Mitosol vesicles depicted as cpm 1 were not detected. The radiolabeled products (cpm 2) have not remained in the area corresponding to intact vesicles (fractions 3 - 9) but instead have been found over the entire gradient, and particularly in material corresponding to membrane sheets on the bottom and the top of the gradient (fractions 1, 2 and 17, respectively). The experiment depicted in Figure 5 demonstrated to us that LCL detected in IMM is involved in the opening of CL-bedecked Mitosol vesicles while incorporating their membrane and releasing their cargo.

In continuation, we have searched for the further support to the concept of intramitochondrial delivery of cytosolic proteins with an intend to explain the presence and derivation of the phospholipases that generated LPG in OMM and LCL in IMM. Particular attention was given to identify the PG- and CL-specific PLAs. Thus, the cytosol was subjected to fractionation according to molecular weight and the obtained fraction analyzed for the enzymatic activity toward ER-mitochondrial vesicles and mitochondrial membranes. As illustrated in Figure 6, the examination of cytosolic activity toward mitochondrial membrane lipids afforded two fractions with PG-specific activity. This activity was also revealed in the OMM following fusion with ER-mitochondrial vesicles [5]. But, neither ER-mitochondria specific vesicles, nor IMM incubation with cytosolic PLA produced LPG in their membranes. Moreover, the analyses of the cytosolic fractions revealed that cytosol was free of PLA with CL-specific activity. The analysis of the lipolytic activity revealed that 3 fractions (lane $1-3$ ) shown in Figure 6 had PG-specific PLA $\mathrm{A}_{2}$ activity, but the CL's PLA specificity in the isolated fractions or whole cytosol was not detected.

Based on CL-specific PLA activity demonstrated previously in Mitosol [5], we tend to advocate that the activity reflected in Mitosol was transported into 


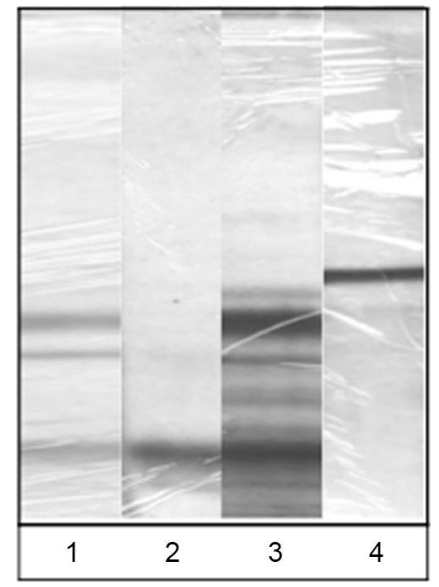

Figure 6. Cytosol derived and partially purified PG phospholipase(s) depicted in lanes 1 3 , the lane 4 represent $66 \mathrm{kD}$ molecular marker. As determined by incubation of palmitate labeled ER-mitochondrial transport vesicles, OMM, Mitosol vesicles and IMM substrates with the isolated cytosolic fractions the lipolytic activity was only displayed toward degradation OMMs PG by generating labeled LPG [5]. However, neither fraction displayed lipolytic activity toward PG present in ER-mitochondrial vesicles, Mitosol vesicles, IMM or CL in Mitosol vesicles and IMM.

intramitochondrial space as the cargo of either OMM or IMM-specific ERtransport vesicles. The Mitosol contained enzyme(s) release palmitate residue from CL [5] which suggests that it may be the one involved in transacylation of IMM lipids. The fact that activity was demonstrated in Mitosol where the vesicles remain intact until their fusion with IMM, the PLA ${ }_{2}$ activity demonstrated in the cytosol could not contribute to CL degradation even if CL-specific enzyme was in cytosol and in Mitosol vesicles cargo. This argues in favor of argument that CL-specific PLA 2 activity is delivered in the ER-mitochondrial cargo and released to Mitosol upon their fusion with OMM. Further study is essential to determine whether the CL hydrolyzing enzyme released to Mitosol from ERmitochondrial vesicles is carried in OMM or in IMM specific transporters.

Thus far, the obtained results allowed us to deduce that ER-transport vesicles directed to mitochondria contain not just those restoring OMM, but also IMM-specific vesicular transporters, both of which initially fuse with OMM, and then upon synthesis of CL, the transporters dedicated for IMM, separate from OMM and fuse with IMM. Furthermore, the IMM-assigned transport vesicles must contain the membrane integrated CL synthase, the protein which upon fusion with OMM faces Mitosol, is responsible for CL synthesis and reforming vesicles dedicated for IMM.

As determined earlier, the OMM membranes are practically free of $\mathrm{CL}$, whereas IMM are enriched in CL that, as documented by using ER-derived radiolabeled vesicles, originated from radiolabeled lipids representing ER-mitochondriaspecific transporters. Further evidence supporting the fact that CL-synthase is transferred exclusively to IMM has been obtained by using purified OMM as the source of CL synthase. The results demonstrated that OMM membranes, as oppose to IMM, could not generate $\mathrm{CL}$, thus substantiating our contention that the 
enzyme was intercalated into IMM membranes only. These results infer that IMM-specific transport vesicles are responsible for the restitution of IMM, and transport of cytosolic cargo for matrix.

The presence of LCL and possibly LLCL in IMM membrane suggests that PLA enzymes are part of Mitosol and/or matrix. To ascertain existence of a such activity on the vesicles and the mitochondrial membranes, the appearance of partially deacylated phospholipids following incubation with their in situ surrounding medium was determined. (This approach was necessary since PLAs activity released labeled fatty acids and the products were not identifiable or discernable). As shown in Figure 7 (lane ER ves), the mitochondria-specific transport vesicles were not susceptible to cytosolic PLAs and remained free of lysolipids, while the OMM as demonstrated earlier [5] and depicted here (lane OMM) contained LPG which upon further incubation with cytosol was reacylated,

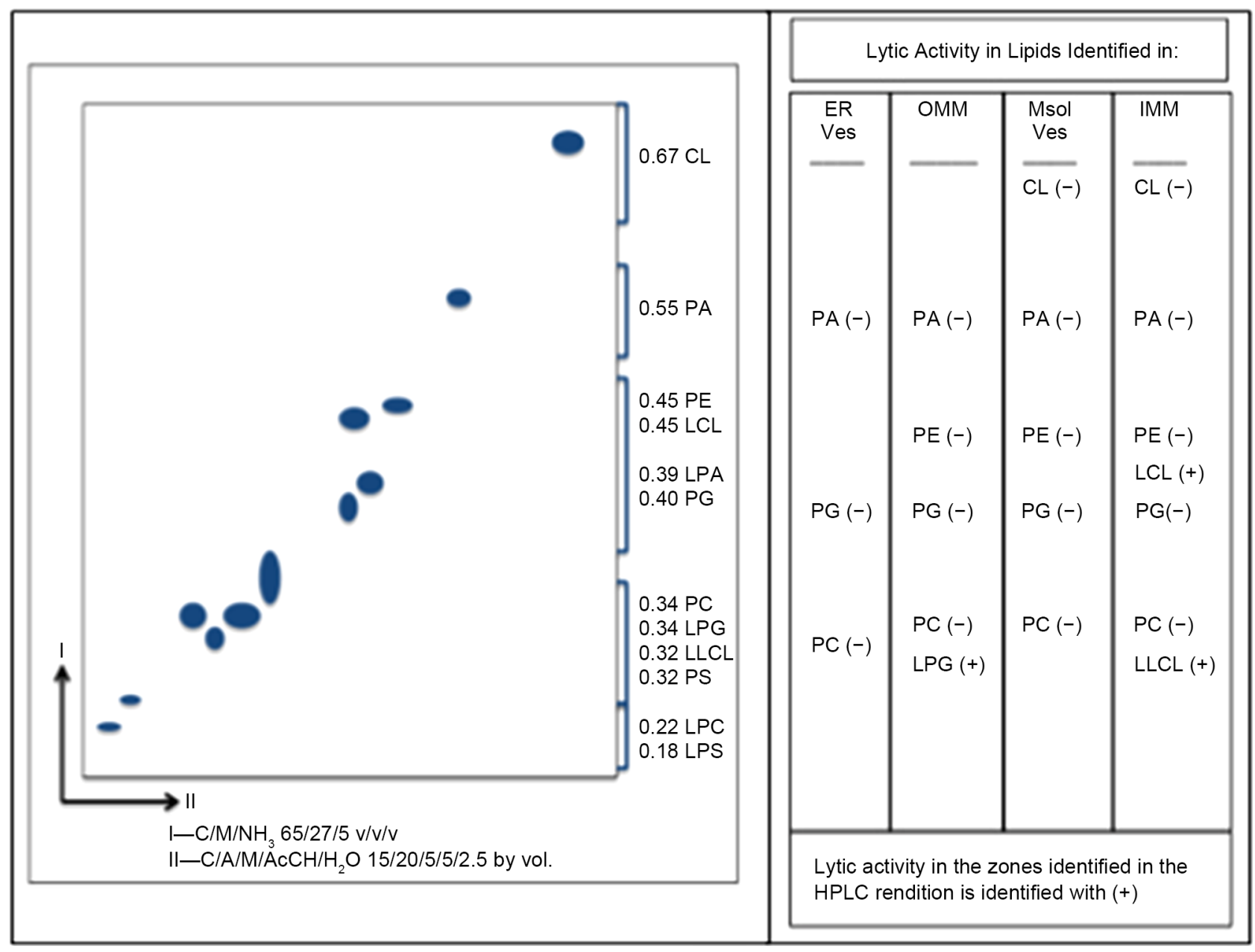

Figure 7. Identification of partially deacylated (lytic) lipids in membranes of the ER-mitochondrial vesicles (ERves), OMM, Mitosol vesicles (Msol ves) and IMM. Neither ER nor Mitosol vesicles contained PG- or CL-derived lysophospholipids. As demonstrated by determining lytic activity in the zones corresponding to the entire spectrum of lipids isolated from the mitochondria-restoring vesicles and the membranes, the lytic activity was found in OMM (LPG +) and in IMM corresponding to LCL (LCL + ) and LLCL (LLCL +). Although assigned lytic activity to LLCL co-chromatographs in the same zone as LPG (OMM fraction), the fact that PG-specific PLAs are not present in Mitosol allows us to suggest that this is the LLCL generated through CL-specific PLA identified in Mitosol [5]. 
meaning that OMM's LPG was facing the cytosolic environment. Similarly to ER-mitochondrial vesicles, the Mitosol vesicles were free of lysolipids (lane mit ves). On the other hand, the analysis of the IMM has shown presence of two PLA-generated product, the LCL and LLCL (lane IMM). Thus, Mitosol vesicles were not affected by CL-specific PLA during incubation in Mitosol, just as ER-mitochondrial vesicles were not susceptible to PG-specific PLA during incubation in cytosol. Evidently, the specific PLA enzymes gained their activity upon association with IMM and OMM, respectively. This type of activation has been demonstrated in our earlier investigations which revealed that PI3P-specific $\mathrm{PLA}_{2}$ was activated upon association with the apical epithelial membrane, the process that generated LPI3P, afforded fusion of apical membrane-specific vesicles and release vesicular cargo [43] [46] [49]. These aforementioned findings on vesicles resistance to cytosolic and mitosolic PLA enzymes provide us with further hints as to the concept of "cell orderly" sequence of events that determine when specific restoration processes are or can take place. As determined in our studies, and multiplicity of other investigations, the cell is equipped with numerous PLAs, yet their action is not causing premature cell demise, but produces harmonious restoration of its parts by rigorously controlled process of the association with acceptor membrane and promoting vesicles' opening.

Thus, in departure from suggested protein import machineries of others, but based on combination of the results supporting the ER-initiated mitochondrial membranes synthesis and their specific inclusion in OMM and then in IMM, we advocate with confidence that mitochondrial restitution is accomplished by first; formation of ER-derived OMM- and IMM-specific transport vesicles that incorporate into OMM, restore OMM and deliver ER-generated cargo to Mitosol, followed by CL synthesis-induced invagination of OMM contained but IMMspecific membrane, while retaining cytosolic proteins associated and interacting with outer leaflet of IMM-specific membrane fragments. Notably, this is the only possible path that can execute delivery of the copious cytosol-synthesized mitochondrial proteins into intramitochondrial matrix without contaminating $\mathrm{Mi}$ tosol.

As documented so far, the cytosol provides phospholipids-specific phospholipase activities and thus represent the major criterion in recognition of the vesicles carrying organelle-specific cargo. Again, in the study presented herein, the results document the reliance of the mitochondrial membranes restitution on the activity of cytosolic PG-specific PLA. This is yet another confirmation that the ER-originating mitochondrial vesicles are specific and the major machineries that restore mitochondrial organelle, and at the same time provide cytosolcontained proteins and substrates for the mitochondria-specific anabolic processes.

In continuation of the study on transit of proteins from ER and cytosol to mitochondrial organelle we must determine whether the LCL and LLCL in IMM are generated through CL-specific PLA activity delivered by ER OMM- or IMMspecific vesicles. It will be highly desirable to determine whether intermitochon- 
drial CL-specific PLA functions on the same principle as the cytosolic PLAs involved in vesicles fusion by being activated upon an association with particular membrane site that is to fuse the incoming vesicle and acquire new membrane installment. Indeed, that would demonstrate that PLAs are the major cellular code, the cipher that programs, rules and regulates organellar and cellular renewal and conformity.

\section{Discussion}

Our findings regarding lipid function in membranes biogenesis allow us to conclude that the currently proposed modes of transport, emphasizing the role of protein while marginalizing the impact of lipids on organelles identity, thwart the principle of the specificity of the organelles restoration concept. While the unique characteristics of membranes are achieved either by posttranslational modification within Golgi, or mitochondria, the ER-initiated make-up reflects the combinatorial task of protein and lipid components that provide cues on the specificity of their destination.

The unilateral paradigm of the studies portraying cellular restitution as the continuous inclusion of singly released ER-generated proteins [14] [15] [16] [50] [51] [52] [53] into organelles fails to explain vesicular transport to Golgi, and just the same struggle is encountered to justify the mitochondrial import [10] [29] [30] [31] [32] [33]. The destined to fail, the ill-conceived installment of the irrelevant combinations of lipids, not conforming to organelle make-up, into artifactual combination of proteins derails experimental mimicry of the in vivo systems [28] [34]. Similarly, the idea of tunnel-like spaces that would allow entrance of cytosolic proteins to mitochondrial inner space, the Mitosol and beyond, shows great limitations due to haphazard of unproven hit-or-miss specificity for the organelle's site and function as well as fails to explain import of the cytosolic proteins to matrix [23] [28] [32] [40] [54]. Without framework of the organelle lipids, the combinational complexity of the in vitro created structures, the translocases, their unshielded and unrestrained forms within utilized medium, and undocumented specificity of their integration within mitochondrial membranes, evoke learned judgment that their insertion and functional capacity will be inconsistent, highly unstable, and influenced by changes in the experiment-created microenvironments [35].

As the feasibility of transport model in form of translocases is arguable [29] [30], the certainty of our concept is supported by recognizing that transport of abundant structural components to Golgi, or about 1200 proteins to mitochondria from genome-controlled products residing in ER and in cytosol, is achieved through the organelles-specific vesicular assemblies of proteins and lipids intercalated into organelle-designated membranes [1] [2] [5] [7] [25] [36] [43] [45].

Based on all facets embracing the intracellular transport, we reason that vesicles, their destination, and vesicular membranes' complexity is rendered in ER by genome initiated signals that activate cotranslational synthesis of lipid scaffold to intercalate specific membrane proteins dedicated for the organellar site. It 
is such a membrane framework that endorses faultless multidirectional cellular transport activity and determines the final location and mode of delivery of the precise organelle-targeted products. Past of ER initiation, the efficient trafficking of the precursors is promoted by the cytosolic factors and organelle specific targeting elements which are indispensible for the delivery of the ER-assembled components to Golgi or the mitochondrial receptors, staged at their membranes respectively [1] [2] [5] [25] [42].

Based on the results of the previously investigated Golgi transport pathway and the findings on mitochondria-specific transport vesicles fusing with OMM, the search drove us to identification of the transitory elements intended for IMM restitution. Thus, we progressed with a concept that inner contents of mitochondria separating OMM from IMM is a mitochondrial equivalent of cytosol, hence Mitosol, and as such serves as the medium to modify and deliver ERpreassembled structures to IMM. Our further investigation of Mitosol uncovered the presence of entities that displayed the same physical characteristics as cytosolic transporters targeted for Golgi and mitochondria. Just as cytosolderived 150,000 g sediment, the Mitosolic residue upon gradient fractionation afforded fraction consistent with transport vesicles. As demonstrated, this fraction carried labeled PC when $\left[{ }^{14} \mathrm{C}\right]$ choline tracer was introduced during the synthesis of ER transport vesicles, whereas with $\left[{ }^{3} \mathrm{H}\right]$ palmitate the entire spectrum of ER-mitochondrial vesicles phospholipids enriched by the presence of CL derived from ER labeled mitochondrial vesicles lipid precursors was detected. In addition, but in contrast to ER-mitochondrial transporters, the Mitosol-derived fraction contained proteins synthesized and present in the cytosol.

The identification of lipid-containing entities in Mitosol, that have similar physical assets as transport vesicles isolated from cytosol with ER assembled cargo, but instead containing cytosolic proteins load, allowed us to suggest that they represent mitochondrial transporters dedicated to restore IMM and deliver the cytosol synthesized protein to matrix. Their lipid compositional assets enriched with CL-synthase product, the CL, were indicative of the IMM-dedicated vesicles. After all, the vesicles which remain stationary part of OMM have not generated CL in the outer membrane. Hence, in our opinion, the dependence between protein and lipid make-up illustrated here by CL-synthase and its lipid product on Mitosol-identified vesicles, allows us to conclude that ER to mitochondria transport relies on two specific transport vesicles, one dedicated to restore the OMM and Mitosol, and the other to restore IMM and deliver the cytosolic protein to matrix.

In principle, the mitochondrial transport path, just as Golgi conduit, has counterpart attributes that control the original design of the ER-initiated cellular transporters. Just as in Golgi pathway, the ER-mitochondrial vesicles, firstly all fuse with OMM to rebuilt outer membrane and release ER-synthesized cargo to Mitosol. Then, the IMM-aimed transporters while part of OMM continuum undergo posttranslational Mitosol-induced CL synthesis and severance from OMM. Since the previous investigations have shown that CL is exclusively 
present in IMM, we furthered our conclusion to advocate that mitochondrial vesicles that originated in ER are delivered via OMM to IMM to rebuild the intramitochondrial membrane. We contend that our evidence on intramitochondrial residence of $\mathrm{CL}$ and the presence of cytosolic proteins in the Mitosol-identified vesicles provide strong argument for the presence of two types of ER-assembled mitochondrial vesicles. The OMM-specific mitochondria-directed vesicles correct the size and refurbish the outer membrane, while the IMM-specific transport vesicles that first fuse with OMM and release the ER-synthesized cargo to Mitosol, upon Mitosol-initiated synthesis of PG and CL and growth of inner leaflet, promote inward curling (involution) of the membrane, closure of the CL-enriched IMM specific membrane over the cytosol proteins that associated with them during OMM residency and the release from OMM to Mitosol. Consequently, we hypothesize, that OMM remains CL-free, whereas CL-embellished membrane fragments reformed into Mitosol vesicles are enabled to contact with IMM, go through CL-assisted fusion with inner membrane and deliver the cytosol-synthesized protein cargo to matrix.

Such a mode of transport eliminates ambiguous delivery of substrates, distributes specific compounds to OMM, Mitosol, IMM and matrix, and generates explicit restitution of the membranes. In this scenario, both genome produced mitochondria-specific proteins derived from ER and those synthesized in the cytosol are transferred to the specific sites in highly precise progression of the organelle's membranes restoration and transport of the intraorganellar substrates that define mitochondrial matrix function. Such preciseness and proficiency of the process responsible for transport of approximately $1000-1200$ proteins to and within organelle is vital [29] [55], and through the fundamental logic, disfavors possibility of a single protein import or cocktails made up of proteins of varied function and destination [28] [29] [32].

In this view, the principle of the intimate link between protein and lipid assemblies that we have uncovered for Golgi-restorative pathway, is mirrored in restitution of mitochondria. How we could have derived with such a conclusion? Firstly and as discussed in the introductory statement, it is not plausible to achieve the precision and consistency of membrane proteins exact arrays intercalation to within pre-constructed lipid assemblies at unrestricted posttranslational stage. The only meticulous and genome-defined process of protein intercalation can be achieved through cotranslational and time-coordinated membrane-specific lipid synthesis while in ER [1] [2] [5]. The occurrence of the harmonized construction of the ER-produced transporters has been revealed in the Golgi restitution pathway where the presence of three types of restorative vesicles were uncovered; those rebuilding Golgi membranes and thus restoring Golgi potential to execute posttranslational modifications, and the transitory ER transporters dedicated to regenerate endosomes and cell membrane [7]. In Golgidirected pathway, we have demonstrated that endosome- and cell membranededicated vesicles, following modification of their respective cargoes by Golgiresident enzymes, were released from organelle to cytosol, and based on their 
explicit lipid- and protein-make-up were delivered to correct destinations. At the same time, the Golgi restoring vesicles with their organelle-specific enzymes were retained and thus the genuine Golgi membrane components were renewed [7] [36]. The outstanding examples of lipid-specific navigation of Golgi-modified vesicles was demonstrated in the contribution of sphingomyelin (SM) to direct vesicles to endosomes and basolateral cell membrane, whereas intra Golgi assemblies of glycosphingolipids (GSphL) glycans navigated the transporters destination to the apical epithelial cell surfaces. Consequently, the coordinate, genome-controlled synthesis of the cell organelle elements in ER satisfied fidelity of the restitution of Golgi and the processing of transiting vesicles taking place in Golgi pathway.

From the study described herein, it is apparent that similar principle operates in mitochondrial restitution of their membranes and in the delivery of ER- and cytosol-synthesized cargoes to Mitosol and the mitochondrial matrix. Our attention to detailed analysis of the lipid composition of the mitochondrial vesicles assembled in ER, and the lipid processing along the transport pathways, allowed us to define the uniqueness of the IMM transporters and the distinctiveness of the organellar membranes' structure [5]. Without consideration of the lipid attributes of IMM-dedicated vesicles of Mitosol, it would be impossible to demonstrate virtues of the uncompromised specificity of the genome-controlled transport of ER-assembled and cytosol-synthesized protein while retaining complete integrity of restoration of OMM. Initially the PG-regulated vesicles fusion with OMM determined delivery of genomic products assembled in ER and necessary for the entire organelle, but next through intramitochondrial CL synthesis in the fragments dedicated for IMM, the CL-containing membranes with mitochondria-specific cytosol synthesized proteins and substrates are released from OMM to Mitosol. As documented in Figure 4, the cytosol-derived radiolabeled proteins are enclosed in the IMM-aimed vesicular cargo and that clearly demonstrates that proteins synthesized in the cytosol but required in the mitochondrial milieu are delivered via IMM-dedicated vesicular transport.

In the mitochondrial pathways, first the exclusive presence of PG marked ERmitochondrial vesicles, which then through specific posttranslational formation of CL distinguished vesicles dedicated to IMM and deliver cytosolic products to matrix, allowed us to distinguish independent restoration processes of the membranes connected with cytosolic protein transport to matrix. The vesicles whose function is to restore the cell organelles or membrane leaflets fuse such a way that the membranes orientation and conformity remains, while cargo is delivered to the membrane-delimited specific environment. In case of fusion with IMM the mitochondrial matrix is replenished with components of cytosol, while IMM gains membrane with CL-containing leaflet facing Mitosol. Indeed, this construal of IMM rebuilding and CL positioning in IMM agree with our findings that Mitosol enzymes are involved in releasing the sn- 1 substituted palmitate residue of CL present in IMM [5]. If CL was in matrix-facing leaflet, the Mitosolinduced generation of LCL could have not taken place. 
While vesicles fusion necessitates the disruption of biological membranes' continuity, the role of lysolipids in the process, although well established in cells' lysis, has not been pursued, and the presence of phospholipases in the cytosol have not been yet associated with intracellular transport and organelle restitution. Perhaps, the lack of link of multiple cellular phospholipases with the transport specificity stems from unexplored contribution of the specific phospholipids in the process, or preoccupation with pathways emphasizing transport of individual proteins. Our investigation of the fusion event revealed involvement of the cytosolic, lipid-specific phospholipases that, by creating lysophospholipids and localized membrane lysis in the fusing membranes, result in the incorporation of the vesicular membrane into continuum of the organelle's membrane and by the same, release or expose the cargo to the inner organellar environment. In earlier studies, we have demonstrated the involvement of PI-specific phospholipase $A_{2}$ in fusion of vesicles with the apical epithelial cell membrane, and the PC-specific PLA ${ }_{2}$ in ER-vesicles fusion with Golgi [7] [25] [42] [43] [46] [49]. Here, we have purified the PG-specific phospholipase $A_{2}$ contributing to vesicles fusion with OMM. In all cases, the specificity of organelle-dedicated vesicles also controls the affinity of PLA in PL degradation and lysolipids generation. While we do not yet have a complete documentation CL-specific PLAs, we demonstrated that Mitosol, just as cytosol, is involved in lysolipids generation in IMM membrane [5]. Moreover, the lipolytic activity detected in Mitosol is not promoted through the import of cytosolic enzymes. As our investigations demonstrated, the cytosol phospholipases do not manifest activity toward CL degradation. Therefore, we are inclined to suggest that the CL-specific phospholipases are transported via ER-mitochondrial vesicles and activated only upon the association with IMM membrane. This statement is supported by the findings that Mitosol vesicles although having CL in their outer leaflet are not susceptible to the enzyme. It appears that this is the common phenomenon for cytosolic and mitosolic phospholipases which prevents premature destruction of the transport vesicles and the release their cargo to cytosol and/or Mitosol. Both, untoward consequences if phospholipase activity would not require specific association with the membrane of vesicular destination.

In the context of lipid specificity of the isolated cytosolic PLAs whose involvement in vesicular transporters fusion is undeniable, the common denominator in the uncovered processes is the fact that without inclusion of lipids in the proposed protein-exclusive machineries of cellular and organellar restitution, the import, the export, and the organelle function retention will remain unsolved. One monumental argument against the present visualization of protein complexes structure created either independent of lipids or in mixture of lipids which do not resemble particular membrane composition is that such cellular cocktails would never incorporate in specific organelle-linked fashion but would be found in numerous cellular sites without any order or need for their presence. With this in mind, the convictions rooted in presumption that nuclear proteome alone satisfies all functions in organelle performance reflects the unilateral and 
biased vision of cell operation. The cell performance is dictated by interdependent cellular genomes functionality that are expressed in finely tuned protein and lipid interaction and reflected in the specific vesicular trafficking, restoration of the organelles and thus continuation of the cell survival.

Our findings on cellular transport uncovered ER as a common site that initiates generation of highly specific protein and lipid complexes in form of transport vesicles that reach their destination without loosing their transport cargo and their site-specific components. The Golgi-directed transporters provide restitution of the cell components that require posttranslational glycosylation and synthesis of sphingolipids, both attributes gained through Golgidirected modification of proteins and/or lipids core. The other portion of the ER transport products which do not associate with Golgi, restore mitochondria. Their function is as specific as Golgi directed transporters; to restore structures of the organelle membranes and provide Mitosol and the matrix with substrates synthesized in ER and on cytosolic ribosomes. As evident, these tasks are achieved via OMM and IMM-specific mitochondrial vesicles.

The above presented logical conclusion regarding the importance of specific lipids, synthesized in coordinated fashion with the membrane specific protein and transported through membrane-modifying posts, defies the logic of the concept of the retrograde transport [2] [25] [43] [44] [45] [46]. As put forward, the process is not feasible since posttranslationally-modified membrane lipids and proteins do not represent molecular make-up of ER, the place where genome-specific commands are translated into multiplicity of organellar and cellular actions and the interdependence between anabolic and catabolic pathways. These processes demand a distinct delivery of the organellar substrates that are orchestrated by genome and cannot be effectively satisfied by the cumbersome and lipid-excluding mechanisms of proteins trafficking. This can only be fully achieved when protein translation is in precise coordination with synthesis of lipids that both reflect the make up of the organelle [1] [4] [5] [6] [36] [42] [43] [44] [46]. Only then, the functionality of the nuclear genome that is highly dependent on these finely tuned mitochondria-nuclear interactions is attained.

In a long view, the events depicting the biosynthetic and functional aspects of mitochondria restorations, it becomes obvious that the processes utilize two systems, the ER-confined and cytosol-limited protein pools interacting with each other by displaying affinity between membrane integrated protein and those free in cytosol, thus providing controlled deposition of the protein in a correct space and compartment.

Consequently, the genome-impacted and ER-initiated processes provide an independently build blocks of organelle-specific membranes, with organellespecific proteins flawlessly incorporated into organelle-designated assembly of lipids. Hence, at any given cellular signal, the ER-derived transport vesicles directed for the mitochondrial restitution that contain independent sets of vesicles, with proteins necessary for OMM, Mitosol, IMM and matrix restoration, may be impacted in a different way. As we have demonstrated in Golgi pathway where 
alcohol impeded the synthesis of Golgi-restoring vesicles and thus induced disparity in cell rebuilding [3] [7] [36], the independently controlled processes of synthesis of OMM and IMM-directed transport vesicles may alter mitochondrial structure as reflected in the dysfunction and mitochondrial pathologies. As an example, the mitochondrial function, reflected in CL synthesis and modification, may be independently controlled through signals responsible for generation of IMM-specific proteins and by synthesis of the cytosol constituents required for the matrix function. These two events, while combined into assembly of IMM-directed vesicles may be affected by drugs introduced to cytosol, or deprivation of the cytosol-deposited cell metabolites [7] [36] [56]. Thus, modification of IMM-directed vesicles alone may invoke a plethora of undesirable mitochondrial changes that our lipid-guided findings may uncover and lead to corrective strategies.

Our concept, that lipid markers determine the intracellular specific restitution, is consonant with the view that any modification in the ER-synthesized membrane replacements may derail organelle protein composition and function, distort cytosol contribution to mitochondrial import, or alter Golgi and the cell functionality [27] [36] [57].

Considering that all transport vesicles originate from ER and must fuse with the existent organelles to deliver cargo and to renew the membrane components, the nature of the triggering factor that initiates specific fusion events remains of paramount importance. While these aspects of membrane fusion are investigated widely, we have concentrated on phospholipids degrading enzymes and lysis-producing products. As we have shown, the fusion of transport vesicles with Golgi is achieved in the presence of PC-specific phospholipase A2, whereas those restoring apical and basolateral cell membrane are assisted by PIs-specific PLA2s [58]. Indeed, we have demonstrated earlier that in mitochondrial transport, the PG-specific PLA supported fusion of the vesicles with OMM membrane and Mitosol aided in the generation of LCL in IMM [5]. Moreover, in continuation of the investigation on the PLAs involvement in fusion of intracellular transporters with the specific organellar membrane, we have uncovered in the cytosol the presence of PG-specific PLA $\mathrm{A}_{2}$ enzymes. Apparently, these enzymes do not release palmitate from PG and do not generate LCL. Further investigation should determine whether the PG-specific enzymes contribute to the processing of cytosolic cargo destined to matrix. Nonetheless, our current data already imply that PG-specific enzyme remain in the cytosol and their function is to delegate the ER-mitochondrial vesicles to the specific site of the organelle that require restoration.

\section{References}

[1] Slomiany, A., Sano, S., Grabska, M., Yamaki, K. and Slomiany, B.L. (2004) Gastric Mucosal Cell Homeostatic Physiome. Critical Role of ER-Initiated Membrane Restitution in the Fidelity of Cell Function Renewal. Journal of Physiology and Pharmacology, 55, 837-860.

[2] Slomiany, A. and Slomiany, B.L. (2010) Cell Membranes Composition Is Defined in 
ER and Their Restitution Proceeds by En Bloc Fusion of ER Generated Transport Vesicles. Health, 2, 1444-1454. https://doi.org/10.4236/health.2010.212214

[3] Slomiany, A. and Slomiany, B.L. (2011) Transformations of Phosphatidylinositol Phosphates in the Outer and Inner Nuclear Membrane Are Linked to Synthesis and Restitution of Cellular Membranes. Health, 3, 187-199.

https://doi.org/10.4236/health.2011.34035

[4] Slomiany, A., Grabska, M. and Slomiany, B.L. (2006) Homeostatic Restitution of Cell Membranes. Nuclear Membrane Lipid Biogenesis and Transport of Protein from Cytosol to Intranuclear Spaces. International Journal of Biological Sciences, 2, 216-226. https://doi.org/10.7150/ijbs.2.216

[5] Slomiany, A. and Slomiany, B.L. (2012) Phosphatidylglycerol-Containing ERTransport Vesicles Built and Restore Outer Mitochondrial Membrane and Deliver Nuclear DNA Translation Products to Generate Cardiolipin in the Inner Mitochondrial Membrane. Advances in Biological Chemistry, 2, 132-145. https://doi.org/10.4236/abc.2012.22016

[6] Slomiany, A. and Slomiany, B.L. (2013) Homeostatic Cell Cycle and the Origin of Autosomal Vesicles. Advances in Biological Chemistry, 3, 275-287. https://doi.org/10.4236/abc.2013.33031

[7] Slomiany, A. and Slomiany, B.L. (2014) Concluding Steps in Cell Restitution Cycle: ER Transport Vesicles with Sphingolipids in the Outer Leaflet of the Membrane Restore Lysosomes. Advances in Biological Chemistry, 4, 301-321. https://doi.org/10.4236/abc.2014.45036

[8] Gohil, V.M. and Greenberg, M.L. (2009) Mitochondrial Membrane Biogenesis: Phospholipids and Proteins Go Hand in Hand. Journal of Cell Biology, 184, 469472. https://doi.org/10.1083/jcb.200901127

[9] Van Meer, G., Voelker, D.R. and Feigenson, G.W. (2008) Membrane Lipids: Where They Are and How They Behave. Nature Reviews Molecular Cell Biology, 9, 112 124. https://doi.org/10.1038/nrm2330

[10] Houtkooper, R.H. and Vaz, F.M. (2008) Cardiolipin, the Heart of Mitochondrial Metabolism. Cellular and Molecular Life Sciences, 65, 2493-2206. https://doi.org/10.1007/s00018-008-8030-5

[11] Scarpulla, R.C. (2008) Transcriptional Paradigms in Mammalian Mitochondrial Biogenesis and Function. Physiological Reviews, 88, 611-638. https://doi.org/10.1152/physrev.00025.2007

[12] Rampelt, H. and Pfanner, N. (2016) Coordination of Two Genomes by Mitochondrial Translational Plasticity. Cell, 167, 308-310. https://doi.org/10.1016/j.cell.2016.09.042

[13] Rothman, J.E. (1994) Mechanisms of Intracellular Protein Transport. Nature, 372, 55-63. https://doi.org/10.1038/372055a0

[14] Lemmon, M.A. (2008) Membrane Recognition by Phospholipids Binding Proteins. National Review of the Molecular Cell Biology, 9, 99-111. https://doi.org/10.1038/nrm2328

[15] Neupert, H. and Herrmann, J.M. (2007) Translocation of Proteins into Mitochondria. Annual Review of Biochemistry, 76, 723-749. https://doi.org/10.1146/annurev.biochem.76.052705.163409

[16] Becker, T., Gebert, M., Pfanner, N. and van der Lann, M. (2009) Biogenesis of Mitochondrial Membrane Proteins. Current Opinions in Cell Biology, 21, 484-493. https://doi.org/10.1016/j.ceb.2009.04.002

[17] Osman, C., Voelker, D.R. and Langer, T. (2011) Making Heads or Tails of Phospho- 
lipids in Mitochondria. Journal of Cell Biology, 192, 7-16.

https://doi.org/10.1083/jcb.201006159

[18] Claypool, S.M., Octay, Y., Boontheung, P., Loo, J.A. and Koehler, C.M. (2008) Cardiolipin Defines the Interactome of the Major Carrier Protein of Mitochondrial Inner Membrane. Journal of Cell Biology, 182, 937-950.

https://doi.org/10.1083/jcb.200801152

[19] Idone, V., Tam, C. and Andrews, N.W. (2008) Two-Way Traffic on the Road to Plasma Membrane Repair. Trends in Cellular Biology, 18, 552-559. https://doi.org/10.1016/j.tcb.2008.09.001

[20] Sarrl, E., Sicart, A., Lazaro-Dieguez, F. and Egea, G. (2011) Phospholipid Synthesis Participates in the Regulation of Diacylglycerol Required for Membrane Trafficking at Golgi Complex. The Journal of Biological Chemistry, 286, 28632-28643. https://doi.org/10.1074/jbc.M111.267534

[21] Glick, B.S. and Nakano, A. (2009) Membrane Traffic within the Golgi Apparatus. Annual Review of Cell Development Biology, 25, 113-132. https://doi.org/10.1146/annurev.cellbio.24.110707.175421

[22] Kawano, M., Kumagai, K., Nishijima, M. and Handa, K. (2006) Efficient Trafficking of Ceramide from Endoplasmic Reticulum to the Golgi Apparatus Requires VAMP-Associated Protein Interacting FFAT Motif of CERT. The Journal of Biological Chemistry, 281, 30279-30288. https://doi.org/10.1074/jbc.M605032200

[23] Giorgi, C., De Stefani, D., Bononi, A., Rizzuto, R. and Pinton, P. (2009) Structural and Functional Link between the Mitochondrial Network and the Endoplasmic Reticulum. International Journal of Biochemistry and Cell Biology, 41, 1817-1827. https://doi.org/10.1016/j.biocel.2009.04.010

[24] Rowla, A.A. and Voeltz, G.K. (2012] Endoplasmic Reticulum-Mitochondria Contacts: Function of the Junction. Nature Reviews Molecular Cell Biology, 13, 607-615. https://doi.org/10.1038/nrm3440

[25] Slomiany, A., Grabska, M., Piotrowski, E. and Slomiany, B.L. (1994) Intracellular Processes Associated with Vesicular Transport from Endoplasmic Reticulum to Golgi and Exocytosis. Archives of Biochemistry and Biophysics, 310, 247-255. https://doi.org/10.1006/abbi.1994.1164

[26] Klausner, R.D., Donaldson, J.G. and Lippincott-Schwartz, J. (1992) Brefeldin A: Insights into the Control of Membrane Traffic and Organelle Structure. Journal of Cell Biology, 116, 1071-1080. https://doi.org/10.1083/jcb.116.5.1071

[27] Schlame, M. and Ren, M. (2009) The Role of Cardiolipin in Structural Organization of Mitochondrial Membranes. Biochimica et Biophysica Acta, 1788, 2080-2083. https://doi.org/10.1016/j.bbamem.2009.04.019

[28] Gebert, N., Ryan, M.T., Pfanner, N., Wiedemann, N. and Stojanovski, D. (2011) Mitochondrial Protein Import Machineries and Lipids: A Functional Connection. Biochimica et Biophysica Acta, 1808, 1002-1011. https://doi.org/10.1016/j.bbamem.2010.08.003

[29] Schmidt, O., Pfanner, N. and Meisinger, C. (2010) Mitochondrial Protein Import: from Proteomics to Functional Mechanisms, Nature Reviews. Nature Reviews Molecular Cell Biology, 11, 655-667. https://doi.org/10.1038/nrm2959

[30] Chacinska, A., Koehler, C.M., Milenkovic, D., Lithgow, T. and Pfanner, N. (2009) Importing Mitochondrial Proteins: Machineries and Mechanisms. Cell, 138, 628644. https://doi.org/10.1016/j.cell.2009.08.005

[31] Walther, D.M. and Rapaport, D. (2009) Biogenesis of Mitochondrial Outer Membrane Proteins. Biochimica et Biophysica Acta, 1793, 42-51. 
https://doi.org/10.1016/j.bbamcr.2008.04.013

[32] Rehling, P., Model, K., Brandner, K., Kovermann, P., Sickmann, A., Meyer, H.E., KuHlbrandt, W., Wagner, R., Truscott, K.N. and Pfanner, N. (2003) Protein Insertion into Mitochondrial Membrane by Twin-Pore Translocase. Science, 299, 1747-1751. https://doi.org/10.1126/science.1080945

[33] Phillips, M. and Voeltz, G.K. (2015) Structure and Function of ER Membrane Contacts Sites with Other Organelles. Nature Reviews Molecular Cell Biology, 17, 69-92. https://doi.org/10.1038/nrm.2015.8

[34] Quiros, P.M., Mottis, A. and Auwerx, J. (2016) Mitonuclear Communication in Homeostasis and Stress. Nature Reviews Molecular Cell Biology, 17, 213-226. https://doi.org/10.1038/nrm.2016.23

[35] Bonham, K.S., Orzalli, M.H., Hayashi, K., Wolf, A.I., Glanemann, W., Iwasaki, A., Knipe, D.M. and Kagen, J.C. (2014) A Promiscuous Lipid-Binding Protein Diversifies the Subcellular Sites of Toll-Like Receptor Signal Transduction. Cell, 156, 705-716. https://doi.org/10.1016/j.cell.2014.01.019

[36] Slomiany, A. and Slomiany, B.L. (2015) Drug-Induced Disparities in Cell Restoration and Debridement: Ethanol-Triggered Nuclear Misreading of the Restitution Cues. Global Journal of Medical Research, 15, 23-38.

[37] Gebert, N., Joshi, A.S., Kutik, S., Becker, T., McKenzie, M., Guan, X.L., Mooga, V.P., Stroud, D.A., Kulkarni, G., Wenk, M.R., Rehling, P., Meisinger, C., Ryan, M.T., Wiedemann, N., Greenberg, M.L. and Pfanner, N. (2009) Mitochondrial Cardiolipin Involved in Outer-Membrane Biogenesis: Implications for Barth Syndrome. Current Biology, 19, 2133-2139. https://doi.org/10.1016/j.cub.2009.10.074

[38] Labbé, K., Murley, A. and Nunnari, J. (2014) Determinants and Functions of Mitochondrial Behavior. Annual Reviews Cell Development Biology, 30, 357-391.

https://doi.org/10.1146/annurev-cellbio-101011-155756

[39] Faini, M., Beck, R., Wieland, F.AT. and Briggs, J.A.G. (2013) Vesicles Coats: Structure, Function, and General Principle of Assembly. Trends in Cell Biology, 23, 279-288. https://doi.org/10.1016/j.tcb.2013.01.005

[40] Mesmin, B. (2016) Mitochondrial Lipid Transport and Biosynthesis: A Complex Balance. Journal Cell Biology, 201, 6060-6069.

https://doi.org/10.1083/jcb.201606069

[41] Whitelegge, J. (2011) Up-Close with Membrane Lipid-Complexes. Science, 334, 320-321. https://doi.org/10.1126/science.1214084

[42] Slomiany, A. and Slomiany, B.L. (2003) Lipidomic Processes in Homeostatic and LPS-Modified Cell Renewal Cycle. Role of Phosphatidylinositol 3-Kinase Pathway in Biomembrane Synthesis and Restitution of Apical Epithelial Membrane. Journal Physiology and Pharmacology, 54, 533-551.

[43] Slomiany, A., Grzelinska, E., Grabska, M. and Slomiany, B.L. (1992) Intracelular Processes Associated with Glycoprotein Transport and Processing. Archives of Biochemistry and Biophysics, 298, 167-175. https://doi.org/10.1016/0003-9861(92)90108-9

[44] Slomiany, A., Grabska, M., Slomiany, B.A., Grzelinska, E., Morita, M. and Slomiany, B.L. (1993) Intracellular Transport, Organelle Biogenesis and Establishment of Golgi Identity: Impact of Brefeldin A on the Activity of Lipid Synthesizing Enzymes. International Journal of Biochemistry, 25, 891-901. https://doi.org/10.1016/0020-711X(93)90245-A

[45] Slomiany, A., Grzelinska, E., Kasinathan, C., Yamaki, K., Palecz, D. and Slomiany, B.L. (1992) Biogenesis of Endoplasmic Reticulum Transport Vesicles Transferring 
Gastric Apomucin from ER to Golgi. Experimental Cell Research, 201, 1669-1682. https://doi.org/10.1016/0014-4827(92)90280-L

[46] Slomiany, A., Nowak, P., Piotrowski, E. and Slomiany, B.L. (1998) Effect of Ethanol on Intracellular Vesicular Transport from Golgi to the Apical Membrane. Role of Phosphatidylinositol 3-Kinase and Phospholipase A2 in Golgi Transport Vesicles Association and Fusion with the Apical Membrane. Alcohol, Clinical and Experimental Research, 22, 167-175. https://doi.org/10.1111/j.1530-0277.1998.tb03634.x

[47] Buszczak, M., Signer, R.A.J. and Morrisons, J. (2014) Cellular Differences in Protein Synthesis Regulate Tissue Homeostasis. Cell, 159, 242-251. https://doi.org/10.1016/j.cell.2014.09.016

[48] Parsons, D.F., Willims, G.R. and Chance, B. (1966) Characteristics of Isolated and Purified Preparations of the Outer and Inner Membranes of Mitochondria. Annals New York Academy of Sciences, 137, 643-666. https://doi.org/10.1111/j.1749-6632.1966.tb50188.x

[49] Slomiany, A., Grzelinska, E., Yamaki, K. and Slomiany, B.L. (1992) Function of Intracellular Phospholipase $A_{2}$ in Vectorial Transport of Apoproteins from ER to Golgi. International Journal of Biochemistry, 24, 1397-1406. https://doi.org/10.1016/0020-711X(92)90065-9

[50] Cobon, G.S., Crowfoot, D. and Linnane, W. (1974) Biogenesis of Mitochondria. Phospholipid Synthesis in Vitro by Yeast Mitochondrial and Microsomal Fractions. Biochemistry Journal, 144, 265-275. https://doi.org/10.1042/bj1440265

[51] Sudhof, T.C. and Rothman, J.E. (2009) Membrane Fusion: Grappling with SNARE and SM Proteins. Science, 323, 474-477. https://doi.org/10.1126/science.1161748

[52] Van Meer, G., Halter, D., Sprong, H., Somerharju, P. and Egmond, M.R. (2006) ABC Lipid Transporters: Extruders, Flippases, or Flopless Activators? FEBS Letters, 580, 1171-1177. https://doi.org/10.1016/j.febslet.2005.12.019

[53] Mellman, I. and Nelson, W.J. (2009) Coordinated Protein Sorting, Targeting and Distribution in Polarized Cells. National Review of the Molecular Cell Biology, 9, 833-845. https://doi.org/10.1038/nrm2525

[54] Simmen, T., Lynes, E.M., Gesson, K. and Thomas, G. (2010) Oxidative Protein Folding in the Endoplasmic Reticulum: Tight Links to the Mitochondria-Associated Membrane (MAM). Biochimica et Biophysica Acta, 1798, 1465-1473. https://doi.org/10.1016/j.bbamem.2010.04.009

[55] Calvo, S.E. and Mootha, V.K. (2010) The Mitochondrial Proteome and Human Disease. Annual Reviews of Genomics and Human Genetics, 11, 25-44. https://doi.org/10.1146/annurev-genom-082509-141720

[56] Okamoto, K. and Kondo-Okamoto, N. (2012) Mitochondria and Autophagy: Critical Interplay between Two Homeostats. Biochimica et Biophysica Acta, 1820, 595600. https://doi.org/10.1016/j.bbagen.2011.08.001

[57] Nunnari, J. and Suomalainen, A. (2012) Mitochondria: In Sickness and in Health. Cell, 148, 1145-1159. https://doi.org/10.1016/j.cell.2012.02.035

[58] Slomiany, B.L. and Slomiany, A. (2016) Helicobacter pylori-Elicited Induction in Gastric Mucosal Matrix Metalloproteinase-9 (MMP-9) Release Involves ERK-Dependent $\mathrm{cPLA}_{2}$ Activation and Its Recruitment to the Membrane-Localized Rac1/ p38 Complex. Inflammopharmacology, 24, 87-95. https://doi.org/10.1007/s10787-016-0261-8 
Submit or recommend next manuscript to SCIRP and we will provide best service for you:

Accepting pre-submission inquiries through Email, Facebook, LinkedIn, Twitter, etc. A wide selection of journals (inclusive of 9 subjects, more than 200 journals)

Providing 24-hour high-quality service

User-friendly online submission system

Fair and swift peer-review system

Efficient typesetting and proofreading procedure

Display of the result of downloads and visits, as well as the number of cited articles Maximum dissemination of your research work

Submit your manuscript at: http://papersubmission.scirp.org/

Or contact abc@scirp.org 